OPEN

SUBJECT AREAS:

NONLINEAR

PHENOMENA

PHASE TRANSITIONS AND CRITICAL PHENOMENA

Received

6 November 2013

Accepted

29 November 2013

Published

17 December 2013

Correspondence and requests for materials should be addressed to

Z.-G.H. (huangzg@ Izu.edu.cn)

\section{Robustness of chimera states in complex dynamical systems}

\author{
Nan Yao ${ }^{1,2}$, Zi-Gang Huang ${ }^{2,3}$, Ying-Cheng Lai ${ }^{2,4} \&$ Zhi-Gang Zheng
}

'Department of Physics and the Beijing-Hong Kong-Singapore Joint Centre for Nonlinear and Complex Systems (Beijing), Beijing Normal University, Beijing 100875, China, ${ }^{2}$ School of Electrical, Computer and Energy Engineering, Arizona State University, Tempe, AZ 85287, USA, ${ }^{3}$ Lanzhou University, and Institute of Modern Physics of CAS, Lanzhou 730000, China, ${ }^{4}$ Department of Physics, Arizona State University, Tempe, Arizona 85287, USA.

The remarkable phenomenon of chimera state in systems of non-locally coupled, identical oscillators has attracted a great deal of recent theoretical and experimental interests. In such a state, different groups of oscillators can exhibit characteristically distinct types of dynamical behaviors, in spite of identity of the oscillators. But how robust are chimera states against random perturbations to the structure of the underlying network? We address this fundamental issue by studying the effects of random removal of links on the probability for chimera states. Using direct numerical calculations and two independent theoretical approaches, we find that the likelihood of chimera state decreases with the probability of random-link removal. A striking finding is that, even when a large number of links are removed so that chimera states are deemed not possible, in the state space there are generally both coherent and incoherent regions. The regime of chimera state is a particular case in which the oscillators in the coherent region happen to be synchronized or phase-locked.

T he collective dynamics of complex systems are often multifold and much more complicated than the dynamics of individual oscillators. For example, when a large number of oscillators, each possessing very simple dynamics, are coupled together, the collective behaviors of all the oscillators can be highly nontrivial. In the classic Kuramoto network ${ }^{1}$, each oscillator is coupled with every other oscillator - the configuration of a globally coupled network. Each individual oscillator is a simple rotation of certain frequency, and the dynamics of the oscillators differ only in their frequencies. The coupling function is also a simple mathematical function, such as a sinusoidal type of function. For relatively weak coupling the motions of the oscillators are incoherent, due to the heterogeneity in their frequencies, but as the coupling parameter increases through a critical value, coherence can emerge and persist in the form of partial or complete synchronization ${ }^{1-4}$. There is now a large body of literature on synchronization in the Kuramoto network, due to its relevance to many physical, chemical, and biological phenomena ${ }^{5}$.

While the emergence of synchronous behavior as the coupling is strengthened is intuitively reasonable and anticipated in any coupled oscillator network, complex systems often present us with unexpected and sometimes quite surprising phenomena. A striking example is the occurrence of chimera state ${ }^{6-24}$ in non-locally coupled networks of identical oscillators, where different subsets of the oscillators can exhibit completely distinct dynamical behaviors. For example, for a simple form of chimera state, there are two distinct types of behavior among all oscillators in the network: one group of oscillators is nearly perfectly synchronous but the oscillators in the complementary group are completely incoherent. These two types of behaviors emerge as one state of the networked system, in contrast to the phenomenon of multiple coexisting attractors in nonlinear dynamical systems ${ }^{25,26}$, each with its own basin of attraction. In such a system, while the attractors coexist in the phase space, starting from a single initial condition the system approaches asymptotically to only one attractor of certain characteristics, which can be a stable fixed point, a limit cycle, a quasiperiodic state, or even a chaotic attractor, but from the same initial condition the system cannot simultaneously possess more than one of these traits. Signatures of chimera states were first observed from the spatiotemporal evolution of a system of coupled nonlinear oscillators and the phenomenon was named "domain-like spatial structure"27. Chimera states in highly regular and non-locally coupled networks of identical oscillators are thus a quite remarkable type of collective dynamics. We note that nonlocal coupling is relevant to physical systems such as the Josephson-junction arrays ${ }^{28}$ and to chemical oscillators ${ }^{5,6}$ as well.

The paradigmatic setting in which chimera states have been studied theoretically and computationally is that of non-locally coupled phase oscillators ${ }^{6-19}$. A fundamental question is how robust chimera states are with respect to 
perturbations. That is, when the system details deviate from those of the paradigmatic setting or when noise is present, can chimera states still emerge and sustain? In this regard, the issue of noise has been successfully addressed, as chimera states have been experimentally observed in a chemical ${ }^{21}$ and an optical ${ }^{22}$ systems that are intrinsically noisy. An outstanding issue is then how random perturbations to the network structure affect the chimera states. In this paper, we address this structural robustness issue that is fundamental to our understanding of chimera states. In particular, starting from the standard setting of a non-locally coupled array of identical phase oscillators, we remove links systematically but randomly according to the removal probability $p$, and investigate whether and to what extent chimera states can persist as $p$ is increased from zero. For a fixed value of $p$, for an infinite network there are an infinite number of possible configurations. For a realistic network of finite size, the number of configurations can still be extremely large. Due to the randomness in the network structure, the persistence of chimera states can be characterized but in a statistical sense. In particular, given $p$, certain fraction of the network configurations would permit chimera states, while others would not. One can thus define a probability for chimera states, denoted by $F(p)$, where $F(p) \rightarrow 1$ for $p \rightarrow 0$ and in general we expect $F(p)$ to be a decreasing function of $p$. Our extensive computations reveal that chimera states can persist for a range of $p$ values in the sense that $F(p)$ maintains values close to unity even when $p$ is appreciably away from zero, strongly suggesting that the exotic dynamical states are robust with respect to random structural perturbations to the underlying network. We then resort to two independent theoretical approaches, one based on self-consistency and another based on the spectral theory for collective dynamics on networks ${ }^{29,30}$. Both give results that are consistent with those from direct numerical computations. A surprising finding is that, even for relatively large values of $p$ for which a large number of links have been removed and chimera state is deemed unlikely, the division of oscillators into coherent and incoherent groups persists. The commonly recognized chimera state, which occurs for smaller values of $p$, is nothing but a particular case in which the coherent oscillators happen to be synchronized or phase-locked.

\section{Results}

We consider an array of $N$ non-locally coupled phase oscillators, mathematically described by

$$
\frac{\partial \phi\left(x_{i}\right)}{\partial t}=\omega-\frac{2 \pi}{N} \sum_{j=1}^{N} c_{i j} G\left(x_{i}-x_{j}\right) \sin \left[\phi\left(x_{i}\right)-\phi\left(x_{j}\right)+\alpha\right],
$$

where $\phi\left(x_{i}\right)$ is the phase of the $i$ th oscillator at position $x_{i}$. The oscillators are located on a ring, so the range of the space variable is $[-\pi$, $\pi$ ] and the boundary condition is periodic. Since the oscillators are assumed to be identical, the natural frequency and phase parameters, $\omega$ and $\alpha$, respectively, are chosen to be constant and they do not depend on the spatial location of the oscillator. Without loss of generality, we set $\omega=0$ and choose $\alpha \lesssim \pi / 2$. The kernel $G(x)=[1$ $+A \cos (x)] /(2 \pi)$ is a non-negative even function that provides the nonlocal coupling among all the oscillators. The quantity $c_{i j}$ is the $i j$ th element of the $N \times N$ coupling matrix $\mathrm{C}$, where $c_{i j}=1$ if there is a coupling from the $j$ th oscillator to the $i$ th oscillator, and $c_{i j}=0$ indicates the absence of such a coupling. For a fully connected network, we have $c_{i j}=1$ for $i, j=1, \ldots, N$. Random removal of links can be implemented by choosing $c_{i j}=0$ for all possible values of $i$ and $j$ ( $i$ $\neq j$ ) with probability $p$.

For convenience, we introduce a reference frame rotating at the angular frequency $\Omega$, so that the phase variable of any oscillator in this frame can be written as $\theta=\phi-\Omega t$. When a chimera state emerges, $\Omega$ can be chosen to be the average frequency of the oscillators in the coherent subset. To characterize the dynamics, we use the following complex order parameter $Z$ defined $^{8}$ for oscillator $i$ :

$$
Z\left(x_{i}\right) \equiv R\left(x_{i}\right) e^{\mathrm{i} \Theta\left(x_{i}\right)}=\frac{2 \pi}{N} \sum_{j=1}^{N} c_{i j} G\left(x_{i}-x_{j}\right) e^{\mathrm{i} \theta\left(x_{j}\right)},
$$

where the summation on the right side is effectively a weighted average of the quantity $e^{\mathrm{i} \theta}$ over all the neighboring oscillators of $i$ for which $c_{i j}=1$. The quantity $R\left(x_{i}\right)$ thus measures the coherence of oscillator $i$ with respect to its neighbors, and $\Theta$ is the average phase of oscillator $i$. Equation (1) can then be rewritten as

$$
\frac{\partial \theta}{\partial t}=\omega-\Omega-R \sin (\theta-\Theta+\alpha),
$$

where the subscript $i$ has been dropped because the form of Eq. (3) is identical for all the oscillators, and the interactions among the oscillators are through the quantities $R$ and $\Theta$. In a chimera state, those oscillators whose phases are locked at frequency $\Omega$ in the original frame correspond to the stationary solution of Eq. (3) with $\partial \theta / \partial t=0$, which requires $R \geq|\omega-\Omega|$. In contrast, in the same chimera state the drifting oscillators (i.e., those that are not phase-locked) have frequencies differing from $\Omega$. The condition for drifting oscillators is then $R<|\omega-\Omega|$.

For randomized coupling specified by the removal probability $p$, we calculate the time evolution of the system by using three independent approaches. The first is direct numerical integration of Eq. (1) to yield the phase $\phi\left(x_{i}, t\right)$ of each oscillator and its velocity $v\left(x_{i}, t\right)$. The second is to solve the self-consistency equation to obtain the complex order parameter $Z\left(x_{i}\right)$. The third is to derive a partial differential equation (PDE) in the continuum limit to solve for the order parameter. (In Methods, we provide detailed derivations of the selfconsistency equation and of the PDE.) For example, in the self-consistency approach, the contributions of the phase-locked and drifting regions to the order parameter of any given oscillator can be combined into a single summation ${ }^{20}$, and the resulting self-consistency equation is

$$
\begin{aligned}
R\left(x_{i}\right) e^{\mathrm{i} \Theta\left(x_{i}\right)}= & e^{\mathrm{i}(\pi / 2-\alpha)} \times \frac{2 \pi}{N} \sum_{j=1}^{N} c_{i j} G\left(x_{i}-x_{j}\right) e^{\mathrm{i} \Theta\left(x_{j}\right)} \times \\
& \frac{\Delta-\sqrt{\Delta^{2}-R^{2}\left(x_{j}\right)}}{R\left(x_{j}\right)},
\end{aligned}
$$

where $\Delta \equiv \omega-\Omega$ is the difference between the natural frequency of oscillators and the frame frequency. Equation (4) contains three unknowns: $R(x), \Theta(x)$ and $\Delta$, which can be solved by an iterative scheme $^{20}$. As we will demonstrate below, these approaches give essentially the same results.

Examples of chimera states under random removal of links. For $p$ $=0$, the network is fully connected, so chimera state can definitely occur. Figure 1(a) shows the snapshot of the spatial profile of $R(x)$ and $\Delta$ of a typical chimera state obtained from the solutions of the self-consistency equation. The region of coherence in which the oscillators are phase-locked is determined by the condition $R \geq$ $|\Delta|$, while the region of incoherence is characterized by $R<|\Delta|$. The results from direct numerical simulation of Eq. (1) agree with those from the self-consistency equation, as shown in Figs. 1(b-d) for the phase profile $\phi(x)$, the phase-velocity profile $v(x)$, and the order parameter modulus $R(x)$ together with $\Delta$ of the system, respectively. We see that the chimera state possesses a kind of spatial symmetry that reflects the coupling pattern of the oscillators. As we begin to remove the links randomly by increasing the value of $p$ from zero, the spatial symmetry is broken, causing certain chimera state to lose its stability. For example, Figs. $1(\mathrm{e})$ and $(\mathrm{f}-\mathrm{h})$ show, for $p=0.8$, the corresponding results from the self-consistency approach and direct numerical solutions, respectively. We observe the disappearance of the phase-locked region due to the relatively high value of the 

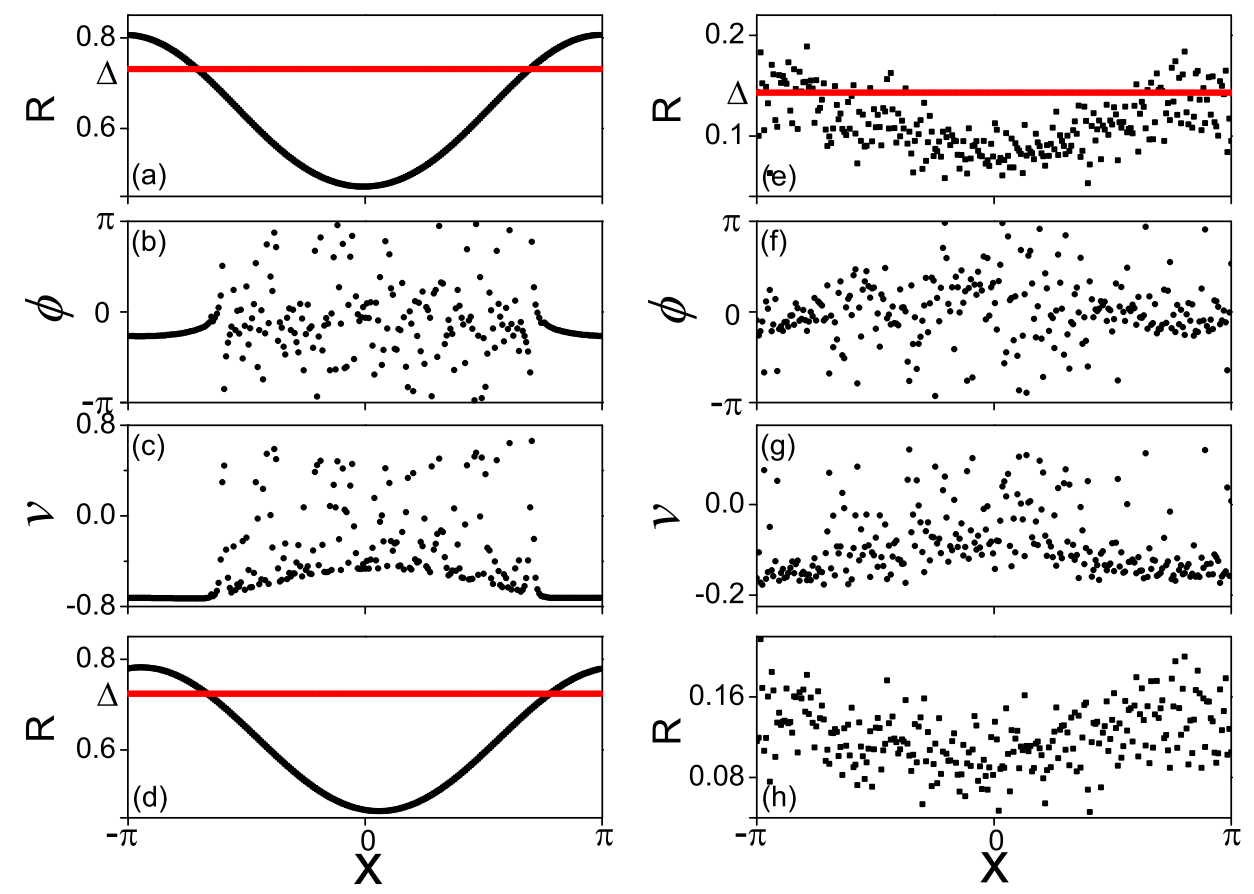

Figure $1 \mid$ Snapshot of the system state in the space. The link removal probabilities are $p=0$ for (a-d) (left column) and $p=0.8$ for (e-h) (right column). (a) $R(x)$ (black solid) obtained from the self-consistency equation (4), where the value of $\Delta$ is indicated (red). (b), (c) Spatial distributions of the phase variable $\phi(x)$ and the phase velocity $v(x)$ obtained from direct numerical integration of Eq. (1), respectively. (d) The corresponding order parameters calculated directly from Eq. (2), where a fixed time step $d t=0.025$ is used, the initial condition is chosen (somewhat arbitrarily) to be $\phi(x)=$ $6 r \exp \left(-0.76 x^{2}\right)$, and $r$ is a random number chosen uniformly from the interval $[-0.5,0.5]$. (e-h) The respective results for $p=0.8$. The parameter $A$ in the kernel function $G(x)$ is set to be 0.995 . Other parameters are $N=256$ and $\alpha=1.39$.

removal probability, signifying destruction of the chimera state. In fact, for large values of $p$, the self-consistency equation for many configurations of the system yields divergent results, indicating the absence of any chimera state. For any $p>0$, the occurrence of chimera state becomes a statistical phenomenon, which can be meaningfully characterized by the probability for chimera state to occur, denoted by $F(p)$, as a function of the removal probability $p$.

To determine, for any coupling configuration under a fixed value of the removal probability $p$, whether a chimera state has occurred, we develop the following criterion. First, we define $e_{v} \equiv$ $|\min [v(x)]| / \varepsilon$, where $v(x)$ is the phase velocity of the oscillator at $x$, which can be obtained numerically from Eq. (1). Next, we say that a chimera state arises if there are more than one but less than $N$ oscillators, each being coherent with its $2 d$ neighbors ( $d$ from the left and $d$ from the right). The set of $2 d+1$ oscillators is regarded to be coherent if the difference in the phase velocities of each and every pair of the $2 d+1$ oscillators is constantly less than $e_{v}$. For any numerically obtained dynamical evolution of the system, we monitor coherence of the oscillators for the final 1000 time steps. A chimera state is deemed to have occurred only if the relevant oscillators remain coherent for all these time steps. This procedure is also applicable to solutions from the self-consistency Eq. (4). In particular, a chimera state is considered to have occurred if there are $2 d+1$ $<N$ oscillators that satisfy the criterion $R>|\Delta|$.

Note that, our numerical criterion to differentiate chimera states is to regard any subset of oscillators as coherent if the difference in the phase velocities of each and every pair of oscillators is constantly less than a small threshold. For chimera state with more than one cluster, this criterion is still applicable because it identifies any subset of coherent oscillators and separates them from the incoherent oscillators. We have observed that, for random removal of links, the chimera state typically contains a single coherent region. Chimera states with multiple clusters of coherent oscillators tend to occur when links are removed in a deterministic fashion. We have tested that our criterion works in all these cases.

Using our criterion for the emergence of chimera states, we calculate, for a systematically increasing set of values of $p$, the frequency $F(p)$ of chimera state from a large number $N_{S}$ of random realizations of the network configuration. Figure 2 shows the results for $N_{S}=$ 1000 and two (somewhat arbitrary) values of the parameter $\varepsilon$ used in the criterion. As anticipated, we observe that $F(p)$ decreases from unity as $p$ is increased from zero. However, for relatively large system size, the probability for chimera state to emerge is still quite large (e.g., greater than 0.8 ) even when the removal probability $p$ exceeds an appreciable value (e.g., 0.2), indicating that chimera states are robust against random perturbations to the network structure. Due to the statistical nature in the calculation of $F(p)$ and the need to choose empirical parameters to detect chimera states, the numerically obtained $F(p)$ curve has a large spread. Nonetheless, the theoretical prediction (open black circles) lies near the middle of the numerical spread, suggesting the validity of our results.

Scaling behaviors. To uncover the possible scaling behaviors in $F(p)$, we focus on the two regimes: one for small values of $p$ in which $F(p)$ begins to decrease from unity, and another for relatively large value of $p$ where $F(p)$ approaches zero. Figures 3(a1) and 3(a2) show the theoretically obtained $F(p)$ curves for two different network sizes. In each case, we identify two critical values, $p_{c 1}$ and $p_{c 2}$, where $F(p)$ starts to decrease from unity as $p$ is increased through $p_{c 1}$ and $F(p)$ approaches zero as $p \rightarrow p_{c 2}$. For $p \gtrsim p_{c 1}$, empirically we have

$$
1-F \propto\left(p-p_{c 1}\right)^{\gamma}
$$

as can be seen from Figs. 3(b1) and 3(b2), where $\gamma>0$ is the algebraic scaling exponent. Similarly, for $p \lesssim p_{c 2}$ and $p \rightarrow p_{c 2}$, we have

$$
F \propto\left(p_{c 2}-p\right)^{\sigma}
$$



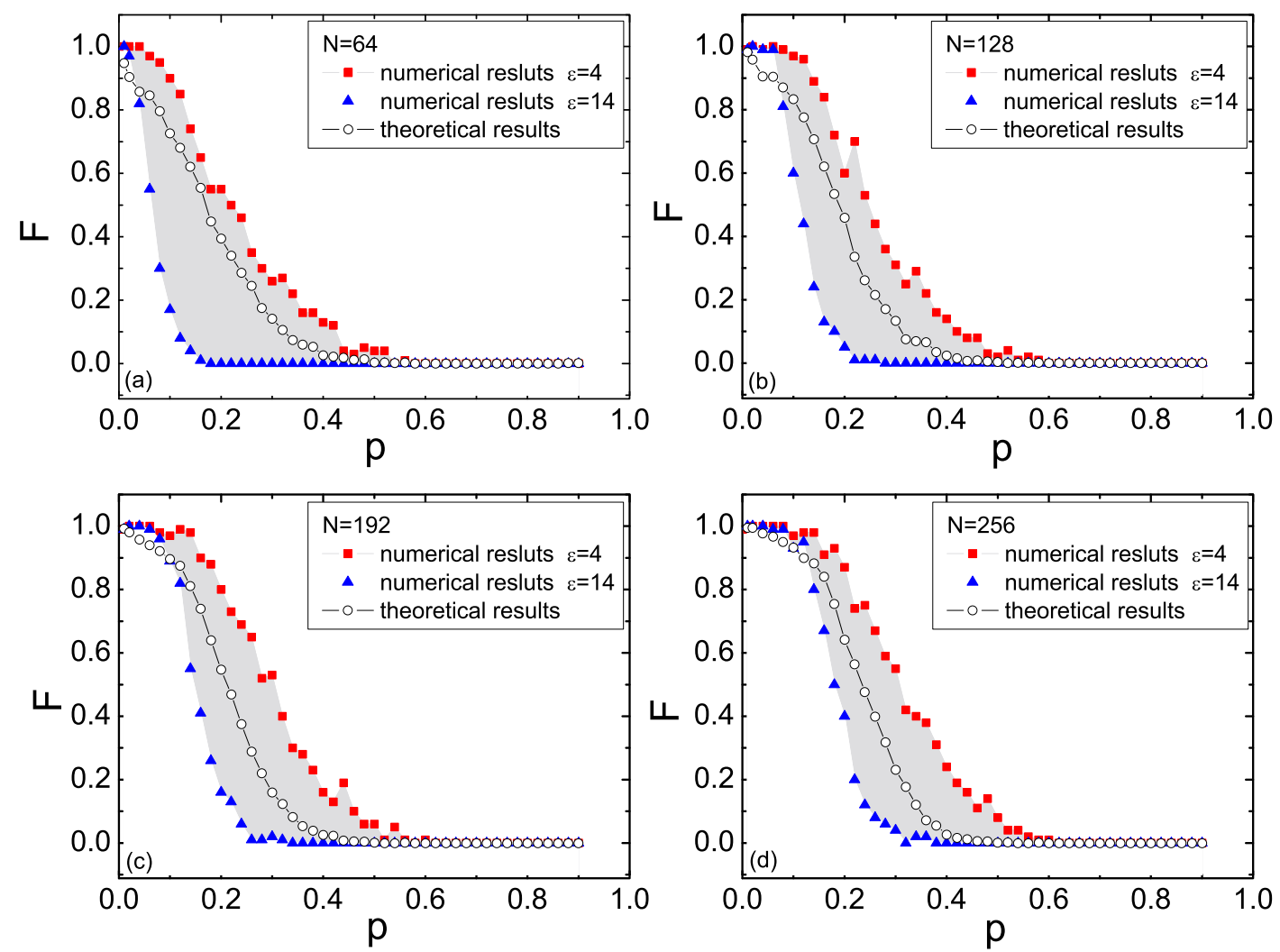

Figure $2 \mid$ Probability $F$ of chimera states. Theoretically and numerically determined probability $F$, or the fraction of network configurations permitting chimera states, as a function of the removal probability $p$. In both approaches, 1000 random network configurations for each fixed value of $p$ are used, where open black circles stand for the result from the self-consistency equation, and red squares and blue triangles indicate the numerical results for $\varepsilon=4$ and $\varepsilon=14$, respectively. The parameters $d$ used to detect the chimera state for different system sizes $N$ are $(\mathrm{a}) d=4$ for $N=64$, (b) $d=8$ for $N=$ 128, (c) $d=12$ for $N=192$, and (d) $d=16$ for $N=256$.

as shown in Figs. 3(c1) and 3(c2), where $\sigma$ is the scaling exponent. The scaling relation (6) can be interpreted, as follows. As $p$ is decreased through $p_{c 2}$, sufficient links among nodes are formed so that chimera state begins to emerge, albeit with low probability. The manner by which the probability of generating chimera state increases is statistically described by the approximately algebraic scaling relation (6).

The behaviors of $F(p)$ as revealed by Figs. 2 and 3 are robust with respect to the system size. To show this, we plot in Fig. 4(a) the theoretically obtained $F(p)$ curves for a number of values of the system size $N$. There is spread among these curves, but it can be significantly reduced by suitable rescaling. For example, we can identify a specific value of $p$, say $p_{0}$, for which the values of $F$ are approximately equal for different system sizes. From Fig. 4(a), we have $F\left(p_{0}\right) \approx 0.36$. If we rescale $p$ according to $p_{0}$ as $p^{\prime}=p / p_{0}$, the resulting $F\left(p^{\prime}\right)$ curves exhibit much smaller spread, as shown in Fig. 4(b).

Spatiotemporal patterns of chimera states. The spatiotemporal behaviors of the oscillator system under random perturbations to the network structure can be assessed by direct numerical integration of the original system Eq. (1). Theoretical insights into the patterns can be obtained by resorting to the continuum limit $N \rightarrow$ $\infty$ to reduce the system to one described by $\mathrm{PDE}^{29,30}$. For the PDE approach, the state of the system is characterized by a probability density function $f(x, \phi, t)$ that satisfies the continuity equation

$$
\frac{\partial f}{\partial t}+\frac{\partial}{\partial \phi}(f v)=0
$$

and can be expressed in terms of Fourier series as

$$
f(x, \phi, t)=\frac{1}{2 \pi}\left\{1+\sum_{n=1}^{\infty}\left[h^{n}(x, t) e^{\text {in } \phi}+\text { c.c. }\right]\right\} .
$$

where "c.c." stands for the complex conjugate of the previous term, and the $n$th coefficient is a function, $h(x, t)$ raised to the $n$th power. The state of the system can then be described by the function $h(x, t)$. Taking into account random perturbations to the links, we obtain the time evolution function of $h\left(x_{i}, t\right)$ associated with the order parameter $Z_{i}$ of the oscillator at $x_{i}$ (see Methods) as

$$
\begin{aligned}
\frac{\partial h\left(x_{i}, t\right)}{\partial t} & =-\mathrm{i} \omega h\left(x_{i}, t\right)+\frac{1}{2}\left[Z_{i}^{*} e^{\mathrm{i} \alpha}-Z_{i} e^{-\mathrm{i} \alpha} h^{2}\left(x_{i}, t\right)\right], \\
Z_{i} & =\frac{2 \pi}{N} \sum_{j=1}^{N} c_{i j} G\left(x_{i}-x_{j}\right) h^{*}\left(x_{j}, t\right) .
\end{aligned}
$$

Figures 5(a1-e1) show, for five values of $p$, the spatiotemporal behavior of $R\left(x_{i}, t\right)$, the module of the order parameter $Z_{i}$ obtained by solving Eq. (9). The corresponding results obtained from direct simulation of Eq. (1) are shown in Figs. 5(a2-e2) for comparison. We observe that, for $p=0$, the spatiotemporal pattern is a kind of breathing chimera state. As $p$ is increased from zero, the period of breathing becomes longer and the value of $R\left(x_{i}, t\right)$ is reduced. From the scaling behavior of $F(p)$ (e.g., Fig. 4), we see that chimera state is not probable for $p \gtrsim 0.4$. However, Figs. $5(\mathrm{~d} 1, \mathrm{~d} 2, \mathrm{e} 1, \mathrm{e} 2)$ reveal that even in this regime there are pronounced spatiotemporal patterns in the system, similar to the breathing chimera state for $p=0$.

In addition to the order parameter $Z$, the complex parameter $h(x, t) \equiv r e^{i \psi}$ in the PDE approach can also be used to characterize the state of any individual oscillator, where $r$ is a measure of the 

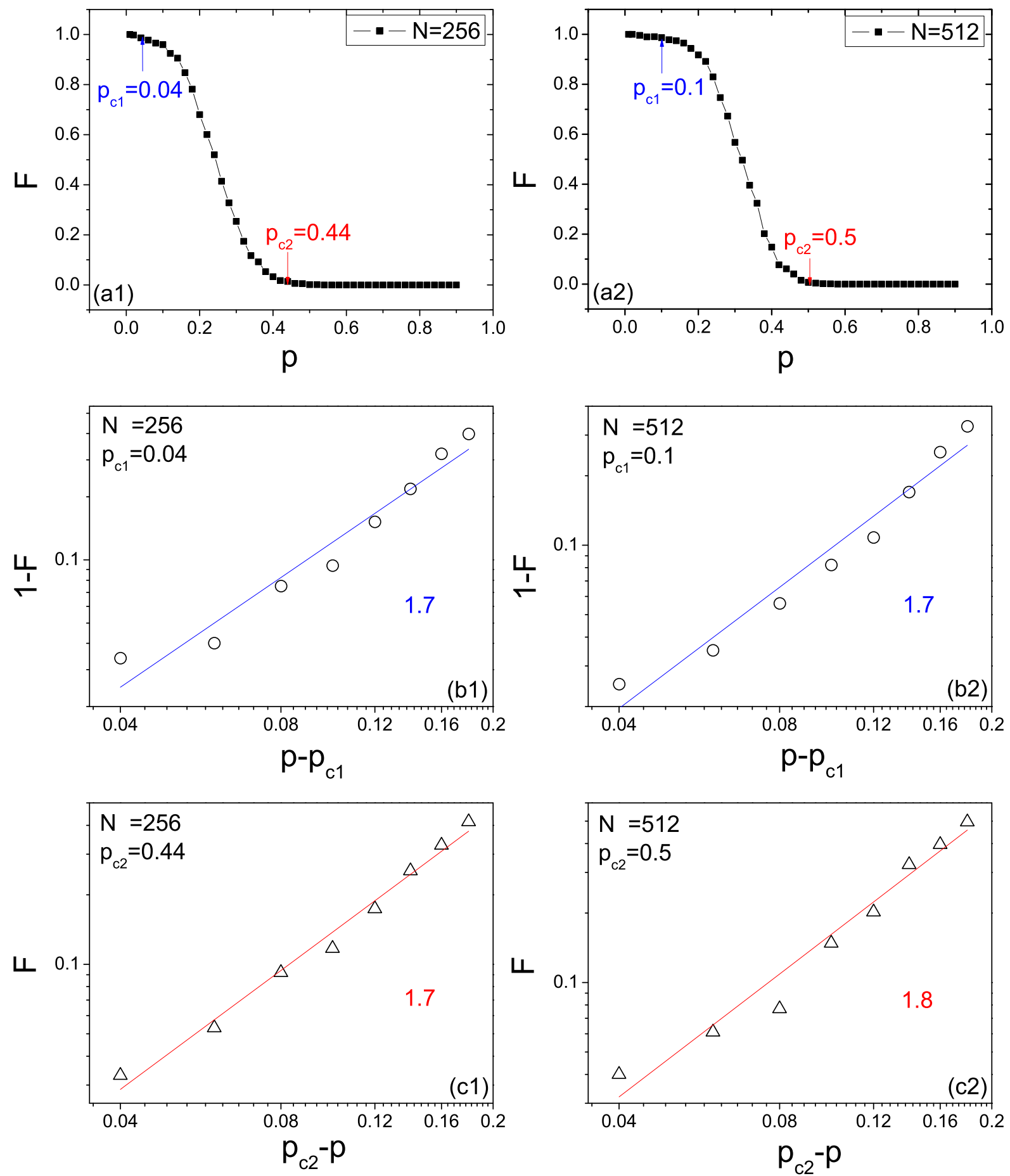

Figure $3 \mid$ Scaling behaviors of the probability of chimera states. (a1), (a2) The probability of chimera states estimated theoretically for two system sizes, $N=256$ and 512, respectively. The critical points $p_{c 1}$ and $p_{c 2}$ for the two systems are indicated by the arrows. The algebraic scaling behaviors of $F$ in the close vicinities of the two points are show in (b1), (b2) and (c1), (c2) on a logarithmic scale, respectively.

sharpness of the probability distribution $f(x, \phi, t)$. In particular, $r=0$ corresponds to a uniform distribution, while $r=1$ signifies the Dirac delta distribution $\delta(\phi-\psi)$. The value $\psi$ gives the phase of $h(x, t)$ at the maximum of $f(x, \phi, t)$. The continuity equation (7) implies that the impact of the oscillators at other spatial locations, as described by the phase-velocity function $v$, may shift the distribution $f(x, \phi, t)$. The trajectories of $h(t)$ in its own complex plane for some representative oscillators are shown in Fig. 6 for the situations where the system exhibits a breathing chimera state (left-hand side for $p=0$ ) and the system is not in any chimera state (right-hand side for $p=0.4$ ). For the two top panels, the oscillator is selected from a region of high coherence, where the trajectory of $h(t)$ moves about the unit circle in the complex plane. For the two bottom panels, the oscillator is from a subset among which the coherence is much weaker. We see that the corresponding trajectories are somewhat random. These results suggest that, for a nonlocally-coupled array of identical oscillators, the self-organized mode characterized by the coexistence of spatially high-coherent and weak-coherent domains, as well as temporally breathing behavior stand out as a general type of spatiotemporal pattern, with or without random structural perturbations. The 



Figure $4 \mid$ Probability of chimera states for different system sizes. (a) For four different values of the system size, $F(p)$ curves calculated by the selfconsistency approach. (b) Rescaled $F\left(p^{\prime}\right)$ curves with reduced spread (see text). The number of network configurations for each fixed value of $p$ is 1000 . Other parameters are $d=4$ for $N=64, d=8$ for $N=128, d=12$ for $N=192$, and $d=16$ for $N=256$.

chimera state is effectively a particular case of the spatiotemporal breathing pattern where the oscillators in the high-coherent domain happen to be synchronized or phase-locked.

\section{Discussion}

Motivated by the growing recent interest in chimera state in nonlocally coupled network of identical oscillators, we address the fundamental issue of robustness of chimera state against random structural perturbations to the network. Using direct numerical simulation and a self-consistency equation, we find that chimera state can persist in a probabilistic sense: the probability of the occurrence of chimera state can be finite even when a large fraction of the links in the networks are removed. The probability to observe chimera state exhibits critical behaviors with the variation in the linkremoval probability.

Utilizing direct numerical computation and an analytic approach based on the PDE model derived in the continuum limit, we study the spatiotemporal pattern of the system of non-locally coupled identical oscillators. Especially, by varying the link-removal probability, we uncover a rather striking phenomenon: regardless of whether chimera state can emerge, the system exhibits a general breathing pattern in its spatiotemporal evolution. Associated with such a pattern, the oscillators in the system can be qualitatively classified into two groups: one group of high coherence and another of weak coherence. The particular breathing pattern stipulates that this division holds even for large link-removal probability where chimera state is ruled out. The implication is that the breathing pattern in the spatiotemporal evolution of the system is general and robust, and chimera state is a particular phenomenon where the oscillators in the highly coherent group happen to be phase synchronized.

Our work thus provides deeper insights into the dynamical origin of chimera state, a phenomenon of continuous interest and subject to intense recent investigation $^{6-24}$.

\section{Methods}

Self-consistency approach. By introducing a complex order parameter depending on space and time as given in Eq. (2), we can rewrite the dynamical equations of the oscillator system as Eq. (3), i.e.,

$$
\frac{\partial \theta}{\partial t}=\omega-\Omega-R \sin (\theta-\Theta+\alpha)
$$

The motion of the oscillator at $x$ can be solved, using the fact that the oscillators with $R(x)>|\omega-\Omega|$ asymptotically approach a stable fixed point $\theta^{*}$ defined implicitly by

$$
\omega-\Omega=R(x) \sin \left[\theta^{*}-\Theta(x)+\alpha\right],
$$

where the oscillators are phase-locked at frequency $\Omega$ in the original frame. To calculate the contribution from the phase-locked oscillators to the order parameter, we note that any fixed point of Eq. (10) must satisfy

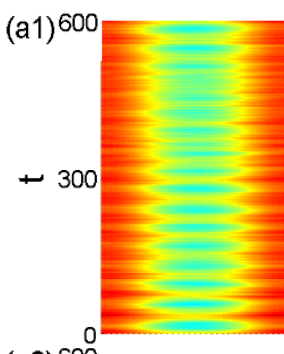

(a2) 600
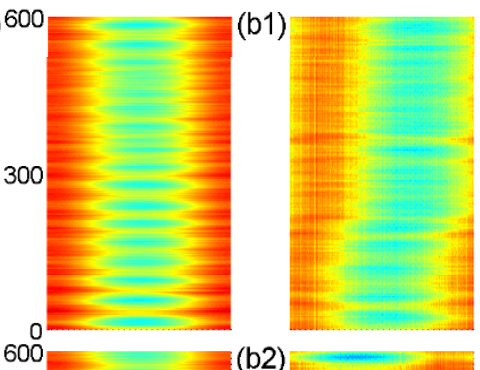

(c1)

(b2)

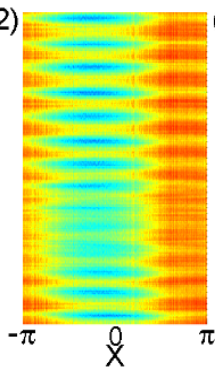

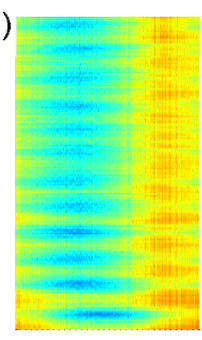



(c2)

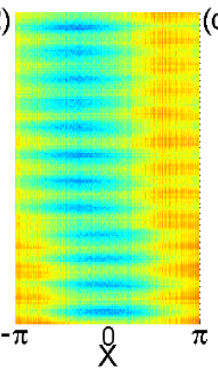

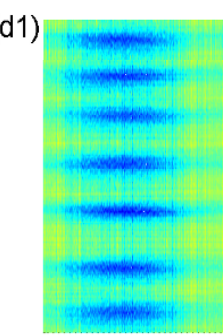

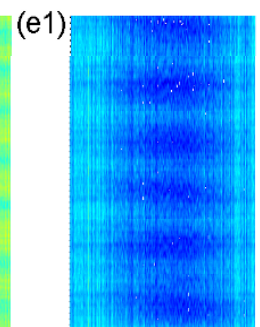

(d2)
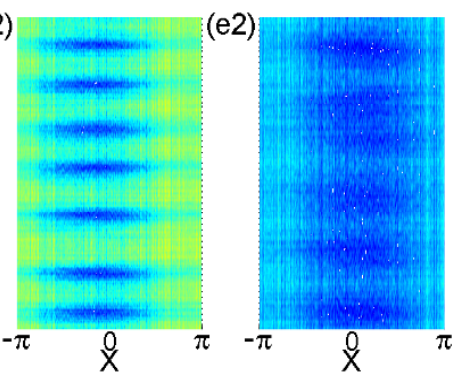

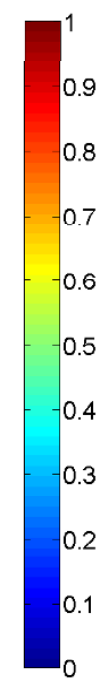

Figure 5 Spatiotemporal evolution of the order parameter. Contour plots representing spatiotemporal evolution of $R(x, t)$ for five values of the link removal probability $p$ (from left to right: $0,0.1,0.2,0.4$, and 0.6 ). The five patterns in the top row are obtained by the PDE in the continuum limit (the PDE approach), and the corresponding patterns in the bottom row are from direct numerical calculations of the original dynamical system. 
(a1)

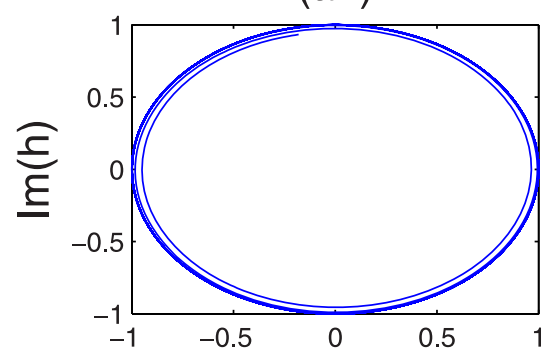

(a2)

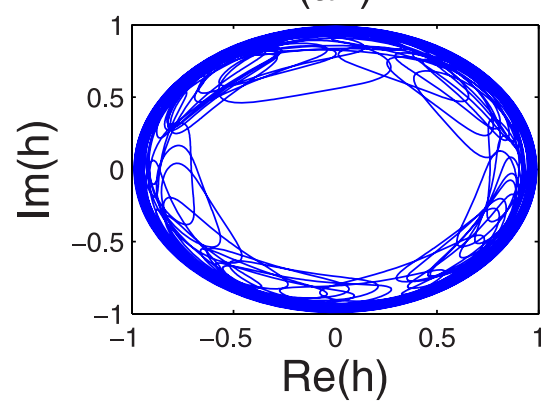

(b1)

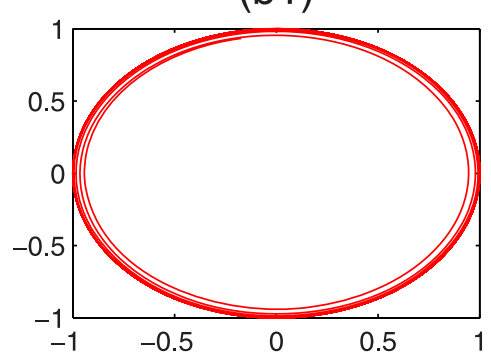

(b2)

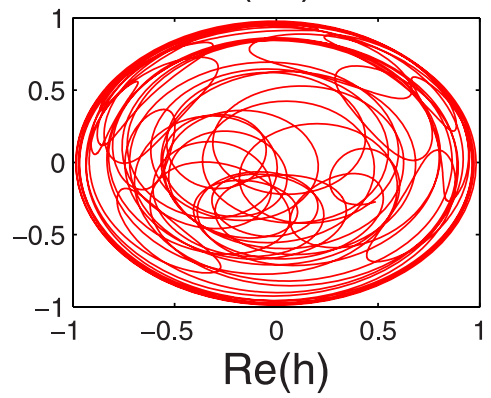

Figure 6 | Temporal behavior of $\boldsymbol{h}(\boldsymbol{t})$ for individual oscillators. Panels (a1) and (b1) correspond to a representative oscillator selected from the region of high coherence, while panels (a2) and (b2) are for an oscillator from the region of weak coherence. For (a1), (a2) and (b1), (b2), the values of $p$ are $p=0$ and 0.4 , respectively.

$$
\sin \left(\theta^{*}-\Theta+\alpha\right)=\frac{\omega-\Omega}{R} \text { and } \cos \left(\theta^{*}-\Theta+\alpha\right)= \pm \frac{\sqrt{R^{2}-(\omega-\Omega)^{2}}}{R},
$$

where the stable fixed point of Eq. (10) corresponds to the plus sign in Eq. (12). Hence, we have

$$
\exp \left[\mathrm{i}\left(\theta^{*}-\Theta+\alpha\right)\right]=\frac{\sqrt{R^{2}-(\omega-\Omega)^{2}}+\mathrm{i}(\omega-\Omega)}{R},
$$

implying that the contribution to the order parameter from the phase-locked oscillators is

$$
\frac{2 \pi}{N} \sum_{j=1}^{N} c_{i j} G\left(x_{i}-x_{j}\right) e^{\mathrm{i} \theta_{j}^{*}}=e^{-\mathrm{i} \alpha} \frac{2 \pi}{N} \sum_{j=1}^{N} c_{i j} G\left(x_{i}-x_{j}\right) e^{\mathrm{i} \Theta_{j}} \times \frac{\sqrt{R_{j}^{2}-(\omega-\Omega)^{2}}+\mathrm{i}(\omega-\Omega)}{R_{j}},
$$

where the summation is taken over the portion of the domain where $R_{j} \geq|\omega-\Omega|$. Hereafter, for convenience, we use subscripts to denote the spatial position of the oscillators, e.g., $R_{j}=R\left(x_{j}\right), \theta_{j}=\theta\left(x_{j}\right), \phi_{j}=\phi\left(x_{j}\right), \Theta_{j}=\Theta\left(x_{j}\right)$, and so on.

The oscillators with $R<|\omega-\Omega|$ drift about the phase circle monotonically, and they distribute themselves according to the following invariant probability density $\rho(\theta)$ :

$$
\rho(\theta)=\frac{\sqrt{(\omega-\Omega)^{2}-R^{2}}}{2 \pi|\omega-\Omega-R \sin (\theta-\Theta+\alpha)|},
$$

where the probability of finding an oscillator near a given value of $\theta$ is inversely proportional to the velocity there so as to make the density invariant. The normalization constant is chosen such that $\int_{-\pi}^{\pi} \rho(\theta) d \theta=1$.

To calculate the contribution to the order parameter from the drifting oscillators, we replace $e^{\mathrm{i} \theta_{j}}$ by its statistical average $\int_{-\pi}^{\pi} e^{\mathrm{i} \theta_{j}} \rho\left(\theta_{j}\right) d \theta_{j}$. Using this approximation in Eq. (15), we have

$$
\int_{-\pi}^{\pi} e^{\mathrm{i} \theta} \rho(\theta) d \theta=\frac{\mathrm{i}}{R} e^{-\mathrm{i}(\alpha-\Theta)}\left[\omega-\Omega-\sqrt{(\omega-\Omega)^{2}-R^{2}}\right] .
$$

The contribution from the drifting oscillators to the order parameter is then given by

$$
\begin{aligned}
& \frac{2 \pi}{N} \sum_{j=1}^{N} c_{i j} G\left(x_{i}-x_{j}\right) \int_{-\pi}^{\pi} e^{\mathrm{i} \theta_{j}} \rho\left(\theta_{j}\right) d \theta_{j}= \\
& \mathrm{i} e^{-\mathrm{i} \alpha} \frac{2 \pi}{N} \sum_{j=1}^{N} c_{i j} G\left(x_{i}-x_{j}\right) e^{\mathrm{i} \Theta_{j}} \times \frac{\omega-\Omega-\sqrt{(\omega-\Omega)^{2}-R_{j}^{2}}}{R_{j}},
\end{aligned}
$$

where the summation is over the complementary part of the domain defined by $R_{j}<$ $|\omega-\Omega|$. Note that the summation is exactly the same as that found earlier in Eq. (14) for the contribution from the phase-locked oscillators, with the only difference being the domains of summation. Since

$$
\sqrt{R^{2}-(\omega-\Omega)^{2}}+\mathrm{i}(\omega-\Omega)=\mathrm{i}\left[\omega-\Omega-\sqrt{(\omega-\Omega)^{2}-R^{2}}\right],
$$

insofar as we choose the branch corresponding to the " $+\mathrm{i}$ " square root of a negative number, the two contributions agree with each other. They can then be combined into one, which is the self-consistency equation derived in?

$$
R_{i} e^{\mathrm{i} \Theta_{i}}=\mathrm{i} e^{-\mathrm{i} \alpha} \frac{2 \pi}{N} \sum_{j=1}^{N} c_{i j} G\left(x_{i}-x_{j}\right) e^{\mathrm{i} \Theta_{j}} \times \frac{\omega-\Omega-\sqrt{(\omega-\Omega)^{2}-R_{j}^{2}}}{R_{j}} .
$$

Letting $\beta=\frac{\pi}{2}-\alpha$ and $\Delta=\omega-\Omega$, we can rewrite the self-consistency equation Eq. (19) as

$$
R_{i} e^{\mathrm{i} \Theta_{i}}=e^{\mathrm{i} \beta} \frac{2 \pi}{N} \sum_{j=1}^{N} c_{i j} G\left(x_{i}-x_{j}\right) e^{\mathrm{i} \Theta_{j}} \times \frac{\Delta-\sqrt{\Delta^{2}-R_{j}^{2}}}{R_{j}} .
$$

The self-consistency equation can be used to solve three unknowns: $R(x), \Theta(x)$ and the real number $\Delta$, which can be accomplished by using an iterative scheme in the functional space. In general, there are two equations [corresponding to the real and imaginary parts of Eq. (20)], and a third equation can be obtained by making the system close. Since Eq. (20) is invariant under the rotation $\Theta(x) \rightarrow \Theta(x)+\Theta_{0}$, we can specify the value of $\Theta(x)$ at any point $x$. A natural choice is to set $\Theta(0)=0$. The resulting three equations can then be solved numerically in a self-consistent manner to yield the three quantities of interest.

PDE approach in the continuum limit. In the continuum limit $N \rightarrow \infty$, the probability density function $f(x, \phi, t)$ satisfies the continuity equation

$$
\frac{\partial f}{\partial t}+\frac{\partial}{\partial \phi}(f v)=0
$$

where

$$
v_{i}=\omega-\frac{2 \pi}{N} \sum_{j=1}^{N} c_{i j} G\left(x_{i}-x_{j}\right) \times \int_{-\pi}^{\pi} d \phi_{j} \sin \left(\phi_{i}-\phi_{j}+\alpha\right) f\left(x_{j}, \phi_{j}, t\right) .
$$

Here the subscripts denote the spatial positions of the oscillators. The order parameter is 


$$
Z_{i} \equiv R_{i} e^{\mathrm{i} \Phi_{i}}=\frac{2 \pi}{N} \sum_{j=1}^{N} c_{i j} G\left(x_{i}-x_{j}\right) \times \int_{-\pi}^{\pi} d \phi_{j} e^{\mathrm{i} \phi_{j}} f\left(x_{j}, \phi_{j}, t\right) .
$$

Equation (22) can be rewritten as

$$
\begin{aligned}
v_{i} & =\omega-\frac{2 \pi}{N} \sum_{j=1}^{N} c_{i j} G\left(x_{i}-x_{j}\right) \times \int_{-\pi}^{\pi} d \phi_{j} \frac{e^{\mathrm{i}\left(\phi_{i}-\phi_{j}+\alpha\right)}-e^{-\mathrm{i}\left(\phi_{i}-\phi_{j}+\alpha\right)}}{2 \mathrm{i}} f\left(x_{j}, \phi_{j}, t\right) \\
& =\omega-\frac{1}{2 \mathrm{i}}\left[e^{\mathrm{i}\left(\phi_{i}+\alpha\right)} \frac{2 \pi}{N} \sum_{j=1}^{N} c_{i j} G\left(x_{i}-x_{j}\right) \times \int_{-\pi}^{\pi} d \phi_{j} e^{-\mathrm{i} \phi_{j}} f\left(x_{j}, \phi_{j}, t\right)\right. \\
& \left.-e^{-\mathrm{i}\left(\phi_{i}+\alpha\right)} \frac{2 \pi}{N} \sum_{j=1}^{N} c_{i j} G\left(x_{i}-x_{j}\right) \times \int_{-\pi}^{\pi} d \phi_{j} e^{\mathrm{i} \phi_{j}} f\left(x_{j}, \phi_{j}, t\right)\right] .
\end{aligned}
$$

Substituting Eq. (23) into Eq. (24), we obtain

$$
v_{i}=\omega-\frac{1}{2 \mathrm{i}}\left[Z_{i}^{*} e^{\mathrm{i}\left(\phi_{i}+\alpha\right)}-Z_{i} e^{-\mathrm{i}\left(\phi_{i}+\alpha\right)}\right] .
$$

Following the treatment in Refs. 29, 30, we express $f(x, \phi, t)$ in terms of the Fourier series:

$$
f(x, \phi, t)=\frac{1}{2 \pi}\left\{1+\sum_{n=1}^{\infty}\left[h(x, t)^{n} e^{\mathrm{in} \phi}+\text { c.c. }\right]\right\},
$$

under the assumption that different harmonics are determined by the corresponding powers of the same function $h(x, t)$. Inserting Eq. (25) and Eq. (26) into the continuity equation (21), we have

$$
\begin{gathered}
\frac{\partial f}{\partial t}=\frac{1}{2 \pi} \sum_{n=1}^{\infty}\left[n h^{n-1} \frac{\partial h}{\partial t} e^{\mathrm{in} \phi}+\text { c.c. }\right], \\
\frac{\partial}{\partial \phi}(f v)=\frac{1}{2 \pi}\left\{\sum_{n=1}^{\infty}\left[\mathrm{i} n h^{n} e^{\mathrm{i} n \phi}+\text { c.c. }\right]\right\} \times\left\{\omega-\frac{1}{2 \mathrm{i}}\left[Z^{*} e^{\mathrm{i}(\phi+\alpha)}-Z e^{-\mathrm{i}(\phi+\alpha)}\right]\right\} \\
+\frac{1}{2 \pi}\left\{1+\sum_{n=1}^{\infty}\left[h^{n} e^{\mathrm{in} \phi}+\text { c.c. }\right]\right\} \times\left\{-\frac{1}{2}\left[Z^{*} e^{\mathrm{i}(\phi+\alpha)}+Z e^{-\mathrm{i}(\phi+\alpha)}\right]\right\} .
\end{gathered}
$$

Comparing the coefficient of the term $e^{\text {in } \phi}$, we obtain

$$
\frac{\partial h}{\partial t}=-\mathrm{i} \omega h+\frac{1}{2}\left[Z^{*} e^{\mathrm{i} \alpha}-Z e^{-\mathrm{i} \alpha} h^{2}\right] .
$$

Substituting Eq. (26) into Eq. (23), we obtain the order parameter as

$Z_{i}=\frac{2 \pi}{N} \sum_{j=1}^{N} c_{i j} G\left(x_{i}-x_{j}\right) \int_{-\pi}^{\pi} d \phi_{j} e^{\mathrm{i} \phi_{j}} \times \frac{1}{2 \pi}\left\{1+\sum_{n=1}^{\infty}\left[h^{n} e^{\mathrm{i} n \phi_{j}}+h^{* n} e^{-\mathrm{i} n \phi_{j}}\right]\right\}$.

The integration of term $e^{\mathrm{i} m \phi}$ is equal to 0 in the interval $[-\pi, \pi]$ when $m$ is an integer except 0 . Equation (30) then becomes

$$
Z_{i}=\frac{2 \pi}{N} \sum_{j=1}^{N} c_{i j} G\left(x_{i}-x_{j}\right) h^{*}\left(x_{j}, t\right)
$$

Equations (29) and (31) can be combined to yield the following equation in terms of $h(x, t)$ and the order parameter:

$$
\begin{aligned}
\frac{\partial h\left(x_{i}, t\right)}{\partial t} & =-\mathrm{i} \omega h\left(x_{i}, t\right)+\frac{1}{2}\left[Z_{i}^{*} e^{\mathrm{i} \alpha}-Z_{i} e^{-\mathrm{i} \alpha} h^{2}\left(x_{i}, t\right)\right], \\
Z_{i} & =\frac{2 \pi}{N} \sum_{j=1}^{N} c_{i j} G\left(x_{i}-x_{j}\right) h^{*}\left(x_{j}, t\right) .
\end{aligned}
$$

From Eq. (32), we can obtain the time evolution of the order parameter.

1. Kuramoto, Y. Chemical Oscillations, Waves and Turbulence (Springer-Verlag, Berlin, 1984).

2. Strogatz, S. H. From Kuramoto to Crawford: Exploring the onset of synchronization in populations of coupled oscillators. Physica D 143, 1 (2000).

3. Restrepo, J. G., Ott, E. \& Hunt, B. R. Onset of synchronization in large networks of coupled oscillators. Phys. Rev. E 71, 036151 (2005).

4. Guan, S.-G., Wang, X.-G., Lai, Y.-C. \& Lai, C. H. Transition to global synchronization in clustered networks. Phys. Rev. E 77, 046211 (2008)

5. Kuramoto, Y. [Where Do We Go from Here?] Nonlinear Dynamics and Chaos [Hogan, S. J. et al. (ed.)] (Institute of Physics, Bristol, 2003).
6. Shima, S. I. \& Kuramoto, Y. Rotating spiral waves with phase-randomized core in nonlocally coupled oscillators. Phys. Rev. E 69, 036213 (2004).

7. Kuramoto, Y. \& Battogtokh, D. Coexistence of coherence and incoherence in nonlocally coupled phase oscillators. Nonlinear Phenom. Complex Syst. 5, 380 (2002).

8. Abrams, D. M. \& Strogatz, S. H. Chimera states for coupled oscillators. Phys. Rev. Lett. 93, 174102 (2004)

9. Abrams, D. M., Mirollo, R., Strogatz, S. H. \& Wiley, D. A. Solvable model for chimera states of coupled oscillators. Phys. Rev. Lett. 101, 084103 (2008).

10. Sethia, G. C., Sen, A. \& Atay, F. M. Clustered chimera states in delay-coupled oscillator systems. Phys. Rev. Lett. 100, 144102 (2008).

11. Sheeba, J. H., Chandrasekar, V. K. \& Lakshmanan, M. Globally clustered chimera states in delay-coupled populations. Phys. Rev. E 79, 055203(R) (2009).

12. Laing, C. R. Chimera states in heterogeneous networks. Chaos 19, 013113 (2009).

13. Laing, C. R. The dynamics of chimera states in heterogeneous Kuramoto networks. Physica D 238, 1569 (2009).

14. Martens, E. A., Laing, C. R. \& Strogatz, S. H. Solvable model of spiral wave chimeras. Phys. Rev. Lett. 104, 044101 (2010).

15. Omel'chenko, O. E., Wolfrum, M. \& Maistrenko, Y. L. Chimera states as chaotic spatiotemporal patterns. Phys. Rev. E 81, 065201(R) (2010).

16. Omelchenko, I., Maistrenko, Y., Hövel, P. \& Schöll, E. Loss of coherence in dynamical networks: spatial chaos and chimera states. Phys. Rev. Lett. 106, 234102 (2011).

17. Wolfrum, M. \& Omel'chenko, O. E. Chimera states are chaotic transients. Phys. Rev. E 84, 015201(R) (2011).

18. Laing, C. R., Rajendran, K. \& Kevrekidis, I. G. Chimeras in random non-complete networks of phase oscillators. Chaos 22, 013132 (2012).

19. Panaggio, M. J. \& Abrams, D. M. Chimera states on a flat torus. Phys. Rev. Lett. 110, 094102 (2013).

20. Abrams, D. M. \& Strogatz, S. H. Chimera states in a ring of nonlocally coupled oscillators. Int. J. Bifurcation Chaos 16, 21 (2006).

21. Tinsley, M. R., Nkomo, S. \& Showalter, K. Chimera and phase-cluster states in populations of coupled chemical oscillators. Nat. Phys. 8, 662-665 (2012).

22. Hagerstrom, A. M. et al. Experimental observation of chimeras in coupled-map lattices. Nat. Phys. 8, 658-661 (2012)

23. Nkomo, S., Tinsley, M. R. \& Showalter, K. Chimera states in populations of nonlocally coupled chemical oscillators. Phys. Rev. Lett. 110, 244102 (2013).

24. Larger, L., Penkovsky, B. \& Maistrenko, Y. Virtual chimera states for delayedfeedback systems. Phys. Rev. Lett. 111, 054103 (2013).

25. Ott, E. Chaos in Dynamical Systems (Cambridge University Press, Cambridge, UK, 2012).

26. Lai, Y.-C. \& Tél, T. Transient Chaos - Complex Dynamics on Finite Time Scales (Springer, New York, 2011).

27. Umberger, D. K., Grebogi, C., Ott, E. \& Afeyan, B. Spatiotemporal dynamics in a dispersively coupled chain of nonlinear oscillators. Phys. Rev. A 39, 4835 (1989).

28. Phillips, J. R., van der Zant, H. S. J., White, J. \& Orlando, T. P. Influence of induced magnetic fields on the static properties of Josephson-junction arrays. Phys. Rev. B 47, 5219 (1993).

29. Ott, E. \& Antonsen, T. M. Low dimensional behavior of large systems of globally coupled oscillators. Chaos 18, 037113 (2008).

30. Ott, E. \& Antonsen, T. M. Long time evolution of phase oscillator systems. Chaos 19, 023117 (2009).

\section{Acknowledgments}

We thank Prof. W.-X. Wang and Prof. L. Huang for helpful discussions. This work was partially supported by AFOSR under Grant No. FA9550-10-1-0083, by NSF under Grant No. CDI-1026710, and the NSF of China under Grants Nos. 11075016 and 11275003, and SPRP of CAS No. XDA03030100. The visit of N.Y. to Arizona State University was sponsored by the State Scholarship Fund of China.

\section{Author contributions}

N.Y., Z.G.H., Y.C.L. and Z.G.Z. devised the research project. N.Y. performed numerical simulations. N.Y., Z.G.H. and Y.C.L. analyzed the results and wrote the paper.

\section{Additional information}

\section{Competing financial interests: The authors declare no competing financial interests.}

How to cite this article: Yao, N., Huang, Z.-G., Lai, Y.-C. \& Zheng, Z.-G. Robustness of chimera states in complex dynamical systems. Sci. Rep. 3, 3522; DOI:10.1038/srep03522 (2013).

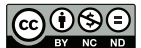

This work is licensed under a Creative Commons Attribution-

NonCommercial-NoDerivs 3.0 Unported license. To view a copy of this license, visit http://creativecommons.org/licenses/by-nc-nd/3.0 